\title{
"Né caccia, né fuga...": techniche di seduzione secondo alcune voci giovani nella letteratura italiana degli anni'90 e 2000
}

Chiara Lombardi

\section{(2) OpenEdition \\ Journals \\ Edizione digitale \\ URL: http://journals.openedition.org/cei/817 \\ DOI: $10.4000 /$ cei.817 \\ ISSN: 2260-779X \\ Editore \\ UGA Éditions/Université Grenoble Alpes}

\section{Edizione cartacea}

Data di pubblicazione: 15 septembre 2006

Paginazione: 81-91

ISBN: 978-2-84310-086-4

ISSN: 1770-9571

Notizia bibliografica digitale

Chiara Lombardi, «"Né caccia, né fuga...": techniche di seduzione secondo alcune voci giovani nella letteratura italiana degli anni'90 e 2000», Cahiers d'études italiennes [Online], 5 | 2006, online dal 15 mars 2008, consultato il 28 mars 2021. URL: http://journals.openedition.org/cei/817 ; DOI: https:// doi.org/10.4000/cei.817 


\author{
"NÉ CACCIA, NÉ FUGA..." \\ TECNICHE DI SEDUZIONE \\ SECONDO ALCUNE VOCI GIOVANI \\ NELLA LETTERATURA ITALIANA DEGLI ANNI '90 E 2000
}

\author{
Chiara Lombardi \\ Università di Torino
}

In occasione di questo convegno sull'evoluzione dell'immagine della seduzione nella narrativa italiana dagli anni '70 ai giorni nostri, ho scelto di parlare di alcuni romanzi scritti tra il 1991 e il $2004,{ }^{1}$ ai quali non viene richiesto di costituire un "canone" di valore, ma solo di essere lo specchio - uno possibile - delle moderne immagini della seduzione e delle sue trasformazioni. In questo contesto, mi è sembrato interessante mettere in luce qualche costante - come il rapporto tra seduzione e scrittura, quest'ultima spesso intesa come forma di compensazione a un desiderio non pienamente realizzato - e anche alcune varianti, specie nel diverso approccio al tema da parte maschile e femminile. La lettura di questi romanzi, inoltre, permette di rispondere a una delle domande che ci sono state rivolte, cioè di considerare come la narrativa, soprattutto negli ultimi anni, tenti di suscitare nel lettore una forma di desiderio, o di dirigerlo verso l'accettazione di nuove forme letterarie che lo contengano. ${ }^{2} \mathrm{D}$ 'altra

1. De Carlo A., Tecniche di seduzione, Milano, Bompiani, 1991; Di noi tre, Milano, Mondadori, 1997; Montrucchio A., Cardiofitness, Venezia, Marsilio, 1998; Gamberale C., Color Lucciola, Venezia, Marsilio, 2001; Elkann A., Una lunga estate, Milano, Bompiani, 2003; Alessandra C, Skill, Torino, Einaudi, 2004; AAVV, Ragazze che dovreste conoscere, Torino, Einaudi, 2004. All'edizione di questi testi si farà riferimento in tutte le citazioni. Molti di questi romanzi - in particolare quelli di De Carlo e della Montrucchio - sono stati tradotti in Francia, Inghilterra, Germania. Cfr. Barenghi M., Oltre il Novecento. Appunti su un decennio di narrativa, Milano, Marcos y Marcos, 1999; Beatrice L., Stesso sangue. DNA di una generazione, Roma, Minimum Fax, 1999; Spinazzola V., Tirature 2000. Romanzi di ogni genere. Dieci modelli a confronto, Milano, Il Saggiatore, 2000.

2. Per una più estesa analisi del desiderio e della seduzione in rapporto alla società e all'arte, $\mathrm{cfr}$. Carotenuto A., Riti e miti della seduzione, Milano, Bompiani, 1994; Zizek S., Il godimento come fattore politico, Milano Cortina, 2001; Dumoulié C., Il desiderio. Storia e analisi di un concetto, Torino, Einaudi, 2002; Senaldi M., Enjoy! Il godimento estetico, Roma, Meltemi, 2003. 
parte, non è sempre facile vedere se e in quale misura l'espressione della seduzione provochi nel lettore uno sconvolgimento dell'"orizzonte d'attesa" tale da preludere a una possibile "risposta estetica". ${ }^{3}$

Lo spunto di questa indagine nella letteratura contemporanea è stato un verso della Gerusalemme Liberata che riporta a uno dei momenti in cui Clorinda e Tancredi si incontrano - e, involontariamente, si seducono prima che l'eroe cristiano uccida la sua avversaria, nonché la donna di cui è innamorato. Nel poema questo momento è preceduto da una serie di altri incontri, dove si svela la forza seduttiva della guerriera pagana e i suoi effetti sull'eroe cristiano. La prima volta Tancredi vede Clorinda a una fonte e se ne innamora, colpito dalla fronte che gli appare come unico elemento di nudità tra l'armatura: "Quivi, a lui d'improvviso una donzella / tutta, fuor che la fronte, armata apparse" (I, 47, 1-2). In un altro incontro, Clorinda va "all'assalto" di Tancredi, il quale, dopo un reciproco scambio di colpi, le rompe i lacci dell'elmo e lascia libere "le chiome dorate al vento sparse" (III, 21, 7). L'eroe resta immobile, si accontenta di guardare, non si decide neanche a dichiararsi. È poi l'arrivo di un guerriero cristiano a rompere l'indugio. Da lui ferita leggermente, Clorinda appare ancora più bella: il "bianco collo" è attraversato da "lievissima piaga" e "i biondi crini / rosseggiaron così d'alquante stille, / come rosseggia l'or che di rubini / per man d'illustre artefice sfaville", III, 30, 3-6). Tancredi ribelle per amore alla sua stessa fede - parte all'inseguimento del soldato cristiano, mentre la guerriera pagana "riman sospesa", li guarda allontanarsi, non le interessa seguirli ("né seguir le cale", III, 31, 3-4), ma si ritira tra quelli della sua parte per ricomparire talvolta per inseguire e difendersi: "or si volge or rivolge, or fugge or fuga, / né si può dir la sua caccia né fuga” (III, 31, 7-8).

Anche per i personaggi maschili e femminili dei romanzi contemporanei che prenderò in considerazione, la seduzione si può dire "né caccia né fuga". Già la figura di Clorinda è ambigua, sia come oggetto di seduzione sia come parte attiva di essa (e ciò è evidente in tutte le corrispondenze metaforiche relative al suo ruolo di guerriera); ma l'ambiguità del personaggio che più o meno consapevolmente seduce diventa un espediente letterario centrale nelle tecniche di seduzione dei personaggi moderni. Inoltre, da un punto di vista formale, come nella Gerusalemme Liberata si

3. Mi riferisco all'estetica della ricezione e agli studi di Iser W., L'atto della lettura. Una teoria della risposta estetica, trad. it. di Granafei R., Bologna, Il Mulino, 1987; Jauss H. R., Estetica della ricezione, trad. it. di Giugliano A., Napoli, Guida, 1988; Perché la storia della letteratura?, trad. it. di Varvaro A., Napoli, Guida, 1989. 
trovano elementi polifonici e trasgressivi all'epica stessa, ${ }^{4}$ così nei romanzi contemporanei incontriamo strutture epiche tradizionali all'interno delle quali prendono vita contenuti moderni e nuove modalità espressive. ${ }^{5} \mathrm{Ad}$ esempio, all'interno di uno schema epico tradizionale, la quest e il duello ampiamente presenti anche nell' Orlando Furioso - diventano filo conduttore possibile di molti processi di seduzione. Nei romanzi contemporanei, essi assorbono le diverse modalità di rappresentazione del desiderio delineando un' imagery scritta da un linguaggio aperto anche ad altre forme artistiche (il cinema, il teatro, la musica, la danza...).

La maggior parte dei romanzi di Andrea De Carlo - come, del resto, quasi tutti i romanzi d'amore - ruotano intorno a una quest, messa in movimento da un incontro folgorante con una ragazza tanto bella quanto difficile e contraddittoria, che irrompe spesso in un momento di calma piatta o di insoddisfazione e rende possibile lo svolgimento della storia con tutti i suoi snodi. Il romanzo Di noi tre si apre con il nome di una ragazza, Misia Mistrani, il cui rilievo spicca già attraverso l'anacoluto: "Misia Mistrani l'ho conosciuta il 12 febbraio del 1978". A partire da quel giorno, il romanzo si svolge per quasi vent'anni intorno alle peripezie di due amici - Livio (la voce narrante, aspirante pittore) e Marco (aspirante regista) - uniti dall'amore per Misia, "questa incredibile ragazza bionda" intravista da Livio la sera della sua laurea in Storia, in un finto pub inglese pieno di fumo, musica, umidità:

Non c'erano molte possibilità di non notarla, anche nella densità estrema dell'aria e nel rumore grattato e martellato ai timpani: aveva quest'aria luminosa, questa naturalezza leggera, questo profilo teso e intelligente quando si girava verso qualcuno dei suoi amici per ascoltare o dire qualcosa o sorridere in un modo così non-affatturato da farmi quasi male al cuore. La sua luce speciale sembrava trasmettersi come un fenomeno elettrico alle persone che le stavano intorno, attraversava lo spazio pieno di gente fino a me che la guardavo a occhiate intermittenti dalla parete opposta; e c'erano altri sguardi nella ressa confusa, non facevano che accentuare il mio senso di mancanza e bisogno, consapevolezza intollerabile dei miei limiti. (p. 10)

4. Osserva Sergio Zatti: "il poema cristiano edificato sulla restaurazione dell'epica antica e, più ambiguamente, sulla contestuale negazione del romance, inaugura in effetti un nuovo tipo di "romanzo", quello dell'introspezione psicologica e della polifonia sentimentale, quello del forte, moderno rilievo dei personaggi" (Zatti S., "Epos, romance, novel: conflitto di codici e trasformazioni di "genere"”, Le immagini della critica, a cura di V. Olivieri, Torino, Bollati Boringhieri, 2003, pp. 135-159, p. 145).

5. Cfr. Barilli R., "L'epica della discoteca», Precisione e imprecisione (Tabucchi, Del Giudice, De Carlo), Torino, Testo e Immagine, 2000, pp. 109-113. 
A questa figura femminile sfuggente e incomprensibile sono consacrati tutto il tempo e lo spazio del romanzo: un tempo finalmente libero, attraverso la narrazione, di dare sfogo a quella tendenza che il professore di Storia rimprovera a Livio durante la discussione della tesi di laurea (" $\mathrm{La}$ storia è prospettiva. Non si può parlare di eventi di sette secoli fa come se fossero successi l'altro ieri e lei ci fosse stato in mezzo", p. 9); uno spazio che si estende da Milano a Firenze, a Roma, dal Brasile alla Colombia, all'Argentina, passando per Zurigo, Londra e Parigi, le Baleari, e perfino per un piccolo paesino dell'Alta Provenza. Qui Misia si ritira per sfuggire ai ritmi delle città e per assecondare lo stereotipo di una vita bucolica e incontaminata (come Angelica tra i pastori...), e qui trascina i personaggi che a loro volta inseguono le intermittenze, le concessioni, le sparizioni, $\mathrm{i}$ colpi di testa (e di scena) creati dalla ragazza, tra stabili occasioni borghesi e desiderio di libertà che non trovano una possibile conciliazione.

Un altrettanto articolato percorso spaziale - molto più rapido, però, anche come durata della narrazione - contraddistingue la quest di Leopoldo, il protagonista del romanzo di Alain Elkann Una lunga estate, da quando incontra Nina:

Una sera di giugno, in Piazza del Popolo, Leopoldo incontrò Nina, una donna bellissima, sensuale, che conosceva da anni, ma di cui sapeva poco. Aveva dei meravigliosi occhi verdi che potevano essere imprevedibilmente dolci, gelidi, intimidenti. Le poche volte in cui l'aveva vista gli era sembrata una gatta, sempre in fuga, misteriosa, abituata a lottare per raggiunge, da sola, una sicurezza che nessuno era mai stato in grado di darle. Uomini si diceva che ne avesse avuti molti, ma ogni volta li aveva lasciati. (p. 7)

Risulta chiaro come gli elementi più sfacciatamente seduttivi, in entrambi i romanzi, siano la bellezza e il mistero, la tendenza alla fuga, che rappresentano il motore dell'azione e, al tempo stesso, il suo ostacolo, in assenza del quale la narrazione non avrebbe ragione d'essere. ${ }^{6}$ Tuttavia, mentre nel testo di De Carlo la seduzione di Misia si svolge per tutta la durata del romanzo senza che la ragazza si conceda al protagonista (ma solo all'amico, Marco, con cui rimane sposata per poco tempo), in Una lunga

6. Il formalista russo Viktor Sklovski parla, per le narrazioni tradizionali, di costruzioni "a gradini", "ad anello", oppure "a nodo". E spiega quello che ci può sembrare ovvio, ma che rappresenta il punto di convergenza tra motivazioni psicologiche e testo: "La descrizione di un amore felice e corrisposto non crea una novella, o anche se la crea, ciò è soltanto perché la si percepisce sullo sfondo tradizionale delle descrizioni di un amore ostacolato". Infatti "la costruzione di una novella richiede non solo una azione, ma anche una contrazione, una cera mancanza di coincidenza. Questo imparenta il "motivo" con il tropo e il calembour" (Sklovski V., "La struttura della novella e del romanzo", I formalisti russi. Teoria della letteratura e metodo critico, a cura di T. Todorov, con pref. di R. Jakobson, Torino, Einaudi, 1968 e 2003, pp. 207-229, pp. 208-209). 
estate Nina si concede quasi subito, provocando così la necessità di nuove e diverse "tecniche di seduzione" per tenere avvinto spasimante e lettore senza generare un'immediata sazietà. All'inizio, le soluzioni adottate sembrano molto facili, sensibili a stereotipi e luoghi comuni. Leopoldo e Nina partono, si godono i piaceri di un soggiorno pieno dei più scontati vizi, indugiano quel tanto che basta per fare sospirare in poche pagine l'inevitabile unione, in un clima da Mille e una notte:

La luna e le stelle, quella notte, erano così luminose da far sembrare tutto incantato. Nina era spensierata e sull'Ape a tre ruote verso casa, dette un bacio sulla guancia a Leopoldo e gli si strinse vicino. Poi scesero dall'Ape, Leopoldo la baciò sulla bocca e lei non oppose resistenza. A casa, salirono sul tetto a guardare le stelle. Era molto tardi quando i loro corpi accaldati e ubriachi si congiunsero. (p. 20)

L'ostacolo, però, sopraggiunge immediatamente:

Verso l'alba Nina disse:

"Mi dispiace, ma oggi arriva Taddeo. Non credo si fermerà a lungo. È geloso e stupido. Questa notte se l'è proprio meritata."

"Che cosa devo fare?"

"Venire a dormire con me."

"E poi?"

"Vedremo." (p. 20)

Taddeo è sposato e, come tutti i personaggi del libro, non ha rapporti stabili; per questo non costituirebbe alcun vero problema per la donna, che sembra quasi disposta a convivere con entrambi per esaltare il proprio fascino. Eppure, se Nina adotta la tecnica di lasciarsi passivamente corteggiare, Leopoldo, invece, si allontana, stacca il cellulare e trascorre alcuni giorni in una specie di rudere abbandonato, consolandosi con il cibo, il vino, con i pescatori e i fumatori di Papastratos e con una sensuale cameriera greca, Irene, che ama ogni notte pur senza dimenticare Nina. E senza cercarla. Questa condizione di malinconico - ma non troppo spiacevole isolamento è interrotto da una lettera di Nina, la quale comunica a Leopoldo la sua partenza per la Corsica, lanciandogli, alla fine, la più invitante e subdola esca (lo fa passare dalla parte del torto e lo lusinga al tempo stesso): "Perché invece di lasciarmi una lettera e fuggire, come se fossimo i protagonisti di un romanzo, non mi hai aspettata? Mi piaceva molto fare l'amore con te! Peccato! Nina” (p. 36).

La lettera di Nina fa scaturire l'inseguimento di Leopoldo lungo un percorso che si svolge parallelo a quello della donna per congiungersi con esso solo alla fine. Dopo la Corsica, infatti, Nina andrà a Parigi diretta a Tangeri, mentre Leopoldo partirà dal Pireo per Roma, arriverà in Corsica 
per poi dirigersi, ripassando un'altra volta per Roma, alla volta di Madrid e poi di Tangeri, dove finalmente rivedrà Nina. Il suo viaggio, però, pur nella ferrea volontà di ritrovare l'innamorata, si nutre di altri indugi più $o$ meno piacevoli. Leopoldo scrive un articolo su Picasso, gusta le specialità di tutti i luoghi, si decide comunque ad inseguirla "forse più per gioco che per amore", oppure perché, come è lui stesso a rivelare: "Nina è una donna bellissima e il mio sentimento per lei non ha limiti di tempo. $\mathrm{O}$ il nostro sarà un amore assoluto o non sarà niente..." (p. 57).

Senza dubbio, quindi, è Leopoldo a creare motivi di indugio, nella volontà di orchestrare quello che la stessa Nina aveva intuito: essere personaggi di un romanzo, sovrapporre arte e vita secondo un ideale romantico-decadente di cui il protagonista è il moderno - e, in fondo, ironico rappresentante per tutto il tempo della storia, fino allo scacco finale e al dissolversi di quel clima di leggerezza tanto rarefatto ed estremo da sfumare con un sorriso e senza drammi. Infatti, la bella e corteggiata Nina befferà, proprio come l'Angelica del Furioso, tutti i suoi corteggiatori, prima sprofondando in un tattico coma (massima esasperazione della sua passività), poi rivelando di essere incinta dell'unico omosessuale del gruppo, il fascinoso fotografo Gérard.

Per molti aspetti, quindi, sembra che il personaggio contemporaneo patisca dello stesso male sofferto dall'artista Raphaël de Valentin in un episodio della Comédie Humaine di Balzac, La Peau de Chagrin, dove il protagonista, insoddisfatto di una vita oscura, in una società consumistica e feroce, riceve dal diavolo una pelle d'asino capace di esaudire tutti i suoi desideri, salvo restringersi ogni volta fino a sparire. Il desiderio, specie quello amoroso e sessuale, consuma con rapida avidità e ferocia la vita, che necessita così di essere ripercorsa e ricomposta attraverso la scrittura. Le "tecniche di seduzione" si confondono con le "tecniche narrative", in un gioco di sospensioni e corrispondenze che sprofonda in una sorta di mise en abîme e che, al tempo stesso, costituisce una delle tentazioni più forti per gli autori contemporanei. Le "insufficienze" del rapporto uomodonna (un sentimento non corrisposto, l'incomunicabilità, la distanza, il tradimento...) suscitano molteplici interrogativi, generano possibili blanks testuali ${ }^{7}$ e rivelano il tentativo dell'ordito narrativo - frutto di quello stesso "pensiero narrativo" che sorge spontaneamente come proiezione e difesa, e vive di tali mancanze ${ }^{8}$ - di colmarli pur senza riuscirci

7. Spesso il testo cerca di ricomporre tali interrogativi senza, però, riuscire a trovare e a dare delle risposte. Cfr. Iser W., Asimmetria tra testo e lettore, in L'atto della lettura. Una teoria della risposta estetica, cit., pp. 241-262, pp. 246 sgg.

8. Cfr. Smorti A., Il pensiero narrativo, Firenze, Giunti, 1994; Smorti A. (a cura di), Il Sé come testo: costruzione delle storie e sviluppo della persona, Firenze, Giunti, 1997. 
completamente (anche perché, se ci riuscisse, il testo perderebbe ogni senso e valore). Ad esempio, come in $D i$ noi tre l'innamoramento avrà come unico effetto quello di tradurre in atto le potenzialità artistiche dei due amici innamorati di Misia, in un altro romanzo di Andrea De Carlo, Tecniche di seduzione, la deludente vicenda narrata si traduce alla fine in quel testo che è appena stato offerto al lettore: "ho pensato che Polidori mi aveva fatto perdere una moglie e una innamorata e il mio primo romanzo, ma in cambio mi aveva lasciato abbastanza sentimenti scoperti da scriverne un altro, questo" (p. 355).

Tale percorso comporta, però, due rischi: 1) un'eccessiva e narcisistica identificazione tra autore e protagonista; 2 ) la produzione di una serie di stereotipi che finiscono con il ripetersi inaridendosi e perdendo suggestioni originali. Ho cercato, proprio per questo motivo, di individuare nel controcanto di alcune scrittrici dello stesso periodo, quindi anche in un cambiamento di punti di vista - alcune possibili varianti a questo leitmotiv. Nel romanzo di Alessandra Montrucchio Cardiofitness, per esempio, si ripresenta la modalità dell'incontro con una persona di sesso opposto, la cui bellezza colpisce e irrompe in un momento di noia, insoddisfazione e squallore. Tra pizze e pomeriggi in centro con le tre amiche battezzate Charlie's Angels, la storia della ventisettenne Stefania si incrocia con quella del quindicenne Stefano intravisto in palestra, spazio descritto come cornice privilegiata della quest e della seduzione:

L'altissimo e magro si aggirava per la zona attrezzi con due manubri in mano, dando la schiena al vetro fumé. Aveva una nuca sottile, e la curva fra il collo e le spalle era la più delicata che Stefania avesse mai visto. (p. 18)

La prima fase della seduzione - tanto più inconsapevole ed eccitante per la giovane età del ragazzo - si gioca su un'immagine baluginante che fonde modelli narrativi tradizionali basati su curiosità e suspence con suggestioni e ritmi cinematografici, e non dimentica l'ironia con cui è scritto tutto il romanzo. La suspence non prevede altri commenti, ma l'azione riprende alla "moviola", concentrandosi sui movimenti e sui discorsi oziosi delle ragazze nello spogliatoio. Solo più avanti si dice che, mentre le amiche passavano accanto alla zona attrezzi, "l'altissimo e magro si sollevò da una panca, posò il bilanciere che aveva usato e guardò Stefania" (p. 20). Il testo prevede un rientro maggiore rispetto a quelli di ogni capoverso, per isolare il cruciale momento che delinea, accanto alle caratteristiche fisiche, un'idea della personalità di Stefano: 
Anche Stefania lo guardò, e lui continuò a fissarla, senza timidezza e senza arroganza, finché non fu lei ad abbassare gli occhi. (p. 20)

A questo punto può sciogliersi la reticenza del testo. Mentre le amiche continuano i loro commenti sull'istruttore, "Stefania pensava agli occhioni stupiti e alla fronte spaziosa dell'altissimo e magro - e peccato che ci fosse un piccolo dettaglio che dava alle sue gambe tremolanti e al suo batticuore un che di allarmante” (p. 20).

La seduzione - concentrata sul "piccolo dettaglio" della nuca (la fronte di Clorinda...) - è iniziata. Ora comincia la quest, anzi, l'“Operazione Tendina" (così, infatti, Stefania ha soprannominato il ragazzino che l'ha colpita, per il modo in cui porta i capelli). Naturalmente, il fatto che sia una donna a condurre il gioco della conquista rovescia - anche se certo non costituisce un elemento di assoluta novità - i rapporti consueti e gli stereotipi della conquista. Ma è soprattutto la differenza di età ad accentuare questo rovesciamento di ruoli, e Stefania si presenta come un moderno Humbert Humbert perso nell'estasi di ogni movimento di Stefano-Lolita.

In seguito agli approcci di Stefania - diretti, coraggiosi, quasi sfacciati, ma che la distinguono dalle noiose e prevedibili ragazzine delle discoteche e dei Mc Donald's - alla fine la conquista ha successo: Stefania e Stefano si baciano, si innamorano, fanno l'amore ed entrano in una specie di film, con tanto di didascalie e di colonna sonora, corrispondente all'intera durata del romanzo. Al termine del racconto, in un torrido luglio torinese, i due innamorati si trovano a discutere proprio sul finale e, inevitabilmente, sul legame tra la loro storia e la scrittura. Stefania, infatti, ha trasformato il loro amore in un romanzo che, a dispetto del soddisfacente andamento della storia e dell'happy ending, ora deve concludersi:

A un certo punto si decide che una storia comincia lì. In quel momento e in quel posto. È più difficile stabilire quando e dove finirà, la storia. Spesso non finisce.

Neanche questa storia finisce.

Ma è una storia felice. Monotona da scrivere, noiosa da leggere, difficile da ricordare, e allora è meglio smettere di raccontarla. (p. 251)

... "Se non si vuole se non si sente se non si sanguina dal desiderio di farlo come si fa come si può scrivere se non si sente se non si vuole se non si ama scrivere quello che scrivere si deve come si...", dice Aleté, la protagonista del romanzo di Chiara Gamberale Color lucciola (p. 71), prima di essere interrotta da Paolo/Orfeo, il ragazzo solitario e malato che lei - dotata del dono maieutico della verità (da cui il nome greco alethé) - dovrebbe fare guarire. Aletè è una verità che prescinde da ogni forma di letteratura, ma, al tempo stesso, sarà colei che, proprio attraverso una seduzione pregna di 
vitalità non soffocata da alcun sapere, guarirà Orfeo restituendogli la gioia di vivere e la possibilità di scrivere, di dare vita al romanzo di Ruggero e Sofia. Aletè, inoltre, rovescia le modalità consuete di rappresentazione della seduzione, prima tra tutte la passività, la tendenza alla menzogna e l'elusività. Eppure, riesce ad accrescere il proprio fascino in modo ancora più efficace:

"Ti avverto che sarà difficile che io riesca a raccontarmi senza metafore."

"Sai una cosa? Metafora è una di quelle parole che sento e ti dirò di più, usi spesso, senza però aver mai avuto voglia di sapere l'esatto significato...” (p. 119)

Aletè, infatti, gioca e seduce con la metafora senza conoscerla, ma con la felice consapevolezza che essa "allontana dalla verità delle cose", e implicitamente la trasferisce nel testo, a sua volta seduttivo perché ricco di simboli quasi magici e di elementi figurali.

Come abbiamo visto, quindi, più o meno tutte queste tecniche di seduzione si manifestano nel corso una quest. Alcune di loro, invece, culminano in un duello. È il caso del romanzo di Alessandra C, Skill, tutto dedicato al gioco virtuale e al percorso di preparazione di un giovane eroe, Skin, prima delle finali del campionato del mondo. Campione indiscusso, superbo e dannato - vive di succo d'arancia e di beveroni ipercaffeinati, sniffa cocaina come se fosse spray per il raffreddore, alterna hamburger e coca-cola ai sashimi di balena, calma la tachicardia con quantità industriali di tavor e si sfoga con le porno-biondine che gli piombano tra le braccia nelle suite degli alberghi di lusso - Skin sarà vinto dalla ragazza di cui è innamorato, Kina, con la quale conduce una sfida virtuale all'ultimo sangue - moderna e capovolta riscrittura, in questa specie di epopea futurista, del duello tra Tancredi e Clorinda:

La mappa ha le sfumature del tardo pomeriggio. Un tramonto sintetico colora le colline di sfondo. [...]

Con tono sensuale Kina sussurra: - Skin, ti piace il mio costumino da squaw? - Poi spara una bordata che mi trancia di netto il braccio sinistro. Quaranta meno.

Entro nel saloon. Mi sposto dietro il bancone del bar e raccatto la Colt. Salgo al primo piano, esco da una finestra e mi lancio. Atterro in piedi. Meno venti per cento punti-vita. Rientro alla porta, Kina è di spalle. Le sparo due colpi alla testa. Frag. [...] Lei ha effettuato il respawn.?

Non la vedo. Devo stare attento e distrarla, mi restano solo quaranta punti-vita.

Passo rasente ai muri.

Non riesco a focalizzare dove si sia appostata, da dove abbia sparato. La traiettoria è precisa, il proiettile mi fora il polpaccio. Meno venti punti. [...]

9. Cioè la "resurrezione" del personaggio all'interno del videogioco, una sorta di "seconda vita". 
Lei mi lascia fare qualche passo in avanti poi spara dritta. Al cuore.

Respawn.

Le do la caccia per tutta la mappa. La vedo sparire, sparire.

Il massacro diventa sistematico.

Dentro e fuori dai fabbricati. Nei sentieri ocra che si tingono di carminio. Rosse le sfumature del tramonto.

Dentro e fuori.

Sono sotto di due frag. Sono in un bagno di sudore. Le tempie mi martellano. Ho la mascella talmente contratta da non riuscire a deglutire.

Silenzio nell'arena silenzio nella mappa.

Il vento artificiale mi ondeggia nei capelli.

Meno cinquanta secondi.

Tempo di gioco.

Prendiamo la mira simultaneamente.

Tempo. Mio.

Spariamo.

La manco.

Mi centra. [...] - Game over! (pp. 170-171)

\section{Le Printemps adorable a perdu son odeur $!^{10}$}

Se consideriamo nel loro complesso questi romanzi - che costituiscono soltanto in minima parte l'orizzonte letterario italiano sul tema della seduzione negli ultimi anni - si può notare come spesso il desiderio prenda forma con tanta efficacia che il lettore non può non esserne contagiato. Pensiamo al momento del primo bacio tra Stefania e il quindicenne Stefano in Cardiofitness:

Stefano dovette interrompersi, perché Stefania lo aveva preso per la nuca e lo stava baciando. Lei. per alcuni istanti, Stefano rimase mummificato e con la bocca chiusa; poi percepì il caldo umido delle labbra di lei, il suo corpo al di là dei vestiti, e proprio quando Stefania stava per lasciarlo le andò incontro, aprì la bocca e la baciò. Lui.

"Ma di quanto sei cresciuto, da quando ti conosco?" gli chiese Stefania non appena si staccarono per riprendere fiato.

"Due o tre centimetri, penso. Perché?"

"Devo stare quasi sulle punte per baciarti, sai? Guarda."

Stefania incollò di nuovo la bocca alla sua, e Stefano si sentì incendiare da una vampata di desiderio e intraprendenza [...]. (p. 115)

oppure al primo incontro d'amore di Roberto e Maria in Tecniche di seduzione:

Maria ha tirato delle tende blu e mi ha guardato, e l'ho abbracciata di nuovo, siamo caduti sul letto e ci siamo rotolati stretti, ci siamo baciati e strusciati come se volessimo mangiarci, impazienti di eliminare le stoffe che ci coprivano e percorrere con le

10. Baudelaire C., Les Fleurs du mal, LXXX. 
mani ogni avvallamento di pelle nuda. E quando ho finito di toglierle tutto di dosso sono rimasto paralizzato a vedere così da vicino le sue forme molto più dolci di come sembravano sotto i vestiti e di come mi ricordavo a teatro: il bianco morbido del suo collo e dei suoi seni e della sua pancia, delle sue cosce e dei polpacci e delle caviglie sottili e dei piedi. [...] Poi ho allungato una mano a carezzarle un seno, e mi sono chinato a baciarglielo, e tutto il tempo ero consapevole del suo sguardo, delle sue labbra dischiuse e del ritmo del suo respiro; del suo odore leggero di miele e di pane appena sfornato e di sudore dolce da leccare [...]. (p. 201)

D'altra parte, ci si deve domandare se una più efficace "risposta estetica" possa essere suscitata nel lettore da testi come questi o da altri meno indulgenti ad assecondare una rappresentazione esplicita e - per certi aspetti prevedibile e stereotipata del desiderio e della seduzione. In questa direzione va, ad esempio, la raccolta Ragazze che dovresti conoscere. The sex anthology, dove, però, la seduzione tra uomo e donna appare annientata in quasi tutti i racconti, a vantaggio di un'idea di morte e di violenza che occupa pressoché tutto l'immaginario possibile del desiderio. Si tratta di "disorientamento" o di gusto perverso di "stupire"? Forse è meglio che domande come questa restino aperte, anche in attesa che, col tempo, si possa guardare a testi così vicini a noi con occhio più obiettivo. 